\title{
Anonymous, neutral, and resolute social choice revisited
}

\author{
Ali I. Ozkes ${ }^{1,2}$ (D) M. Remzi Sanver ${ }^{3}$
}

Received: 28 September 2017 / Accepted: 19 December 2020 / Published online: 11 January 2021 (c) The Author(s), under exclusive licence to Springer-Verlag GmbH, DE part of Springer Nature 2021, corrected publication 2021

\begin{abstract}
We revisit the incompatibility of anonymity and neutrality in single-valued social choice. We first analyze the irresoluteness outlook these two axioms together with Pareto efficiency impose on social choice rules and deliver a method to refine irresolute rules without violating anonymity, neutrality, and efficiency. Next, we propose a weakening of neutrality called consequential neutrality that requires resolute social choice rules to assign each alternative to the same number of profiles. We explore social choice problems in which consequential neutrality resolves impossibilities that stem from the fundamental tension between anonymity, neutrality, and resoluteness.
\end{abstract}

\section{Introduction}

Equal treatment of individuals as well as of alternatives are among the core principles of democratic decision-making. Equal treatment of individuals is usually ensured by the anonymity condition, which requires the social choice to be invariant under renaming individuals. The typical condition to ensure equal treatment

\footnotetext{
We are grateful to Walter Bossert, Denis Cornaz, Fatih Demirkale, Lars Ehlers, Ayça Ebru Giritligil, Jeffrey Hatley, Sean Horan, Hervé Moulin, Jean Lainé, Clemens Puppe, Yves Sprumont, and William Zwicker for helpful discussions. The paper extensively benefited from the thoughtful comments of three anonymous referees to whom we are grateful. Our work is partly supported by the Projects ANR-14-CE24-0007-01, CoCoRICo-CoDec, and IDEX ANR-10-IDEX-0001-02 PSL* MIFID.
}

Ali I. Ozkes

ali.ozkes@wu.ac.at

M. Remzi Sanver

remzi.sanver@dauphine.fr

1 Léonard de Vinci Pôle Universitaire, Research Center, 92916 Paris La Défense, France

2 Wirtschaftsuniversität Wien, Institute for Markets and Strategy, Welthandelsplatz 1, 1020 Vienna, Austria

3 Université Paris-Dauphine, Université PSL, CNRS, LAMSADE, 75016 Paris, France 
of alternatives, on the other hand, is neutrality, which requires the social choice to change in compliance with renaming of alternatives.

The logical incompatibility between anonymity and neutrality while ensuring an untied outcome is among the most well-known results in social choice theory. Moulin $(1980,1991)$ characterizes the sizes of social choice problems that admit anonymous and neutral social choice rules (SCRs) that are resolute, i.e., that choose a unique alternative at any profile. More precisely, a social choice problem with $n$ individuals and $m$ alternatives admits an anonymous, neutral, and resolute SCR if and only if $m$ cannot be written as the sum of some divisors of $n$ that exceed 1 (Moulin 1991). When (Pareto) efficiency is imposed together with anonymity and neutrality, this requirement is strengthened to " $n$ not having a prime divisor less than or equal to $m$ " (Moulin 1980). ${ }^{1}$

How severe is this tension between anonymity and neutrality? Campbell and Kelly (2015) show the rarity of cases where anonymous, neutral, and resolute SCRs exist: when the number of individuals is divisible by at least two distinct primes, only a finite number of social choice problems admit anonymous, neutral, and resolute SCRs. Also, when the number of alternatives exceeds the smallest prime dividing the number of individuals, a resolute SCR is anonymous and neutral only if it chooses alternatives that are in the bottom half of preferences of all individuals. Adding efficiency to anonymity and neutrality restricts the sizes of social choice problems that admit anonymous, neutral, and resolute SCRs even further.

Do these results leave any hope for guaranteeing equal treatment of voters and alternatives for untied collective choice? We reject pessimism by identifying a weakening of neutrality which allows a vast range of possibilities while pandering to a very significant aspect of equal treatment of alternatives. This new condition that we call consequential neutrality requires that all alternatives are chosen at the same number of preference profiles.

For example, with an odd number of individuals, fixing two alternatives $x$ and $y$ and two individuals $i$ and $j$, one can define the resolute SCR that picks the best alternative of individual $i$ when a majority of individuals prefer $x$ to $y$ and otherwise, picks the best alternative of individual $j$. This SCR presents a procedure that depends on the names of the alternatives, hence failing neutrality. On the other hand, being consequentially neutral, it ensures that every alternative is chosen at the same number of preference profiles, thus putting forward an ex-ante fairness property that is more outcome-oriented compared to the classical neutrality approach that entails a more procedure-oriented equal treatment of alternatives.

Recent research on anonymous and neutral social choice mostly focus on rules that assign orderings (instead of alternatives) to preference profiles. Bubboloni and Gori (2014) and Doğan and Giritligil (2015) characterize the sizes of social choice problems for which anonymous and neutral rules that are resolute, i.e., that assign strict social welfare orderings to profiles, exist. Building on the algebraic approach in Bubboloni and Gori (2014), Bubboloni and Gori (2015) propose a weakening of

\footnotetext{
${ }^{1}$ Zwicker (2016) delivers an introduction to the theory of voting where major results regarding anonymity and neutrality are included.
} 
neutrality by assuming that alternatives are divided into sub-classes and requiring equal treatment of alternatives within each sub-class, while alternatives in different sub-classes may be differently treated. They provide necessary and sufficient conditions for the existence of (reversal) symmetric majority rules that satisfy this weakening of neutrality together with a weaker version of anonymity. ${ }^{2}$ Bubboloni and Gori (2016) adopt the aforementioned versions of anonymity and neutrality together with efficiency and characterize when rules that assign orderings to profiles admit a resolute refinement that preserves these properties. Their characterization identifies the necessary and sufficient arithmetical conditions on the sizes of sub-committees and sub-classes. King and Powers (2018), on the other hand, dispense with neutrality altogether and identify a characterization of rules that satisfy anonymity, monotonicity, and cancellation, in the case of two alternatives.

We start by analyzing the structure of irresoluteness imposed by anonymity, neutrality, and efficiency, a previously overlooked matter. We generalize the characterization of Moulin (1980) by completely describing the sizes of unavoidable ties under these conditions (Theorem 2). This generalization paves the way to identifying a method to refine SCRs that are "more irresolute than necessary," while anonymity, efficiency, and neutrality are preserved (Theorem 3).

We then turn to our analysis of consequential neutrality for resolute SCRs. We start with counting the number of resolute SCRs that are neutral and those that are consequentially neutral $(\mathrm{CN})$ as a function of the size of the social choice problem (Theorem 4). An analytical comparison of these two numbers seems beyond reach, so we take a computational approach where we compute the numbers of resolute SCRs in each class for a small set of values of the size of the social choice problem. These numerical exercises that we report on show strong tendencies in the comparison of the numbers of $\mathrm{CN}$ and neutral SCRs, hence we conjecture that the class of resolute SCRs that are $\mathrm{CN}$ is considerably larger than those that are neutral.

Thereafter, we discuss the possibility of refining anonymous, efficient, and neutral SCRs by replacing neutrality with consequential neutrality and deliver a possibility result under certain conditions (Theorem 5). We also identify some cases where these conditions are not satisfied but there exist anonymous, $\mathrm{CN}$, and resolute SCRs (Theorem 6). These positive results do not hold over all conceivable social choice problems: we point to instances where anonymity, consequential neutrality, efficiency, and resoluteness turn out to be incompatible (Theorem 7). These are instances where the incompatibility prevails even without efficiency. However, even in those cases, anonymous, $\mathrm{CN}$, and resolute SCRs exist. In fact, we are able to identify a large class of social choice problems, namely those where the number of alternatives exceeds the number of individuals, for which anonymity, consequential neutrality, and resoluteness are compatible (Theorem 9).

The paper is organized as follows. Section 2 gives basic notation and notions. Section 3 delivers a generalization of the classical result on incompatibility of

\footnotetext{
2 They propose a weaker version of anonymity in a similar way by assuming that individuals are divided into sub-committees and requiring that, within each sub-committee, individuals have equal influence on the collective decision, while people in different sub-committees may enjoy different levels of influence.
} 
anonymity, neutrality, and efficiency with resoluteness and proposes a refinement method towards resoluteness. Section 4 introduces consequential neutrality and presents our more permissive results when it replaces neutrality. Section 5 concludes.

\section{Basic notions and notation}

Writing $\mathbb{N}$ for the set of natural numbers and picking $m, n \in \mathbb{N} \backslash\{1\}$, we conceive a social choice problem as a set $A$ of alternatives with $\# A=m$ and a set $N$ of individuals with $\# N=n$. We refer to $(m, n)$ as the size of the social choice problem $(A, N)$. Writing $\mathcal{L}(X)$ for the set of linear orders, i.e., complete, asymmetric, and transitive binary relations on a given set $X$, let $P_{i} \in \mathcal{L}(A)$ denote the preference of $i \in N{ }^{3}$ An $n$-tuple of such individual preferences indicates a (preference) profile $P_{N} \in \mathcal{L}(A)^{N}$. A social choice rule (SCR) is a mapping $f: \mathcal{L}(A)^{N} \rightarrow \mathcal{A}$, where $\mathcal{A}=2^{A} \backslash\{\varnothing\}$ is the set of non-empty subsets of $A$.

Given any two sets $S$ and $T$, we write $S \subseteq T$ whenever $S$ is a subset of $T$ and $S \subset T$ whenever $S$ is a proper subset of $T$. We let $\left.P_{N}\right|_{B}$ denote the restriction of $P_{N} \in \mathcal{L}(A)^{N}$ to those alternatives in $B \in \mathcal{A}$ so that $\left.P_{N}\right|_{B} \in \mathcal{L}(B)^{N}$ and $\left.x P_{i} y \Longleftrightarrow x P_{i}\right|_{B} y$ for all $x, y \in B$ and $i \in N$. Given any two SCRs $f_{1}$ and $f_{2}$, we say that $f_{2}$ refines $f_{1}$ iff $f_{2}\left(P_{N}\right) \subseteq f_{1}\left(P_{N}\right) \forall P_{N} \in \mathcal{L}(A)^{N}$ and $f_{2}\left(P_{N}^{\prime}\right) \subset f_{1}\left(P_{N}^{\prime}\right)$ for some $P_{N}^{\prime} \in \mathcal{L}(A)^{N}$. An SCR $f$ is resolute whenever $\# f\left(P_{N}\right)=1 \forall P_{N} \in \mathcal{L}(A)^{N}$. For a resolute SCR $f$, we write $f\left(P_{N}\right)=x$ in place of $f\left(P_{N}\right)=\{x\}$.

We now define two equal treatment conditions that are at the core of our analysis. For any non-empty finite set $X$, a permutation on $X$ is a bijection $\sigma: X \leftrightarrow X$. Let $\Sigma_{X}$ be the set of all permutations on $X$. We write, by a slight abuse of notation, $\sigma\left(P_{N}\right)=\left(P_{\sigma(i)}\right)_{i \in N}$ for the profile obtained from $P_{N} \in \mathcal{L}(A)^{N}$ by a permutation $\sigma \in \Sigma_{N}$. An SCR is anonymous iff $f\left(P_{N}\right)=f\left(\sigma\left(P_{N}\right)\right) \forall P_{N} \in \mathcal{L}(A)^{N} \forall \sigma \in \Sigma_{N}$. Again, by an abuse of notation, we write $\sigma\left(P_{i}\right)$ for the preference obtained from $P_{i} \in \mathcal{L}(A)$ by a permutation $\sigma \in \Sigma_{A}$ on $A$, i.e., $x P_{i} y \Longleftrightarrow \sigma(x) \sigma\left(P_{i}\right) \sigma(y) \forall x, y \in A$.

Moreover, we set $\sigma\left(P_{N}\right)=\left(\sigma\left(P_{i}\right)\right)_{i \in N} \forall P_{N} \in \mathcal{L}(A)^{N}$. An SCR is neutral iff $f\left(\sigma\left(P_{N}\right)\right)=\sigma\left(f\left(P_{N}\right)\right) \forall P_{N} \in \mathcal{L}(A)^{N}, \forall x \in A$, and $\forall \sigma \in \Sigma_{A}$.

We close the section by noting that an SCR $f$ is efficient iff given any $P_{N} \in \mathcal{L}(A)^{N}$ and any $x \in f\left(P_{N}\right), \nexists y \in A \backslash\{x\}$ with $y P_{i} x \forall i \in N$.

\section{Anonymous, neutral, and efficient social choice}

Given $k, l \in \mathbb{N}$, we write $k \mid l$ whenever $k$ divides $l$, i.e., $\frac{l}{k} \in \mathbb{N}$, and $k \nmid l$ otherwise. Let $\mathcal{D}(n)=\{k \in \mathbb{N}: k \mid n\}$ be the set of divisors of $n \in \mathbb{N}$ and $\mathcal{D}^{*}(n)=\{k$ is a prime $: k \mid n\} \cup\{1\}$ be the set consisting of prime divisors of $n$ as well as 1 . Thus, $\mathcal{D}^{*}(n) \subseteq \mathcal{D}(n)$ for all $n \in \mathbb{N}$. For any $m, n \in \mathbb{N}$, the set of divisors of $n$ that do not exceed $m$ is denoted $\mathcal{D}_{m}(n)=\{d \in \mathcal{D}(n): d \leq m\}$ and similarly

\footnotetext{
3 So, given any distinct $x, y \in A$ and $P_{i} \in \mathcal{L}(A)$, precisely one of $x P_{i} y$ and $y P_{i} x$ holds. Moreover, $x P_{i} y$ and $y P_{i} z$ implies $x P_{i} z$ for all $x, y, z \in A$ and $P_{i} \in \mathcal{L}(A)$. Finally, $x P_{i} x$ does not hold for any $x \in A$.
} 
$\mathcal{D}_{m}^{*}(n)=\left\{d\right.$ in $\left.\mathcal{D}^{*}(n): d \leq m\right\}$. Again, $\mathcal{D}_{m}^{*}(n) \subseteq \mathcal{D}_{m}(n)$ for all $m, n \in \mathbb{N}$. Imposing $\mathcal{D}_{m}^{*}(n)=\{1\}$ is shown by Moulin (1980) to be a necessary and sufficient condition for the size of a social choice problem to admit an anonymous, neutral, efficient, and resolute SCR.

Theorem 1 (Moulin 1980) A social choice problem $(A, N)$ with size $(m, n)$ admits an anonymous, efficient, neutral, and resolute $S C R$ f if and only if $\mathcal{D}_{m}^{*}(n)=\{1\}$.

The condition $\mathcal{D}_{m}^{*}(n)=\{1\}$ in Theorem 1 can be replaced by $\mathcal{D}_{m}(n)=\{1\}$. ${ }^{4} \mathrm{We}$ will refer to $\mathcal{D}_{m}(n)=\{1\}$ as Condition $\mu(m, n) .^{5}$

Theorem 1 gives a complete picture of the sizes of social choice problems where irresoluteness is inevitable but is silent about the structure of irresoluteness in such cases. To analyze this, we define $K_{f}=\left\{\# f\left(P_{N}\right): P_{N} \in \mathcal{L}(A)^{N}\right\}$ as the irresoluteness outlook of SCR $f$. So for any natural number $k \leq m$, we have $k \in K_{f}$ if and only if there exists a profile to which $f$ assigns a set of $k$ alternatives. ${ }^{6}$

Theorem 2 Take any social choice problem $(A, N)$ with size $(m, n)$.

i. $\quad$ An SCR $f$ is anonymous, efficient, and neutral only if $K_{f} \supseteq \mathcal{D}_{m}(n)$.

ii. There exists an anonymous, efficient, and neutral SCR $f$ with $K_{f}=\mathcal{D}_{m}(n)$.

\section{Proof}

i. Take $d \in \mathcal{D}_{m}(n)$. As $d \in \mathcal{D}(n)$, there exists $t \in \mathbb{N}$ such that $n=d t$. Take any set of alternatives $\left\{x_{1}, \ldots, x_{d}\right\} \subseteq A$ and the partition $\left\{S_{1}, \ldots, S_{d}\right\}$ of $N$ with $S_{1}=\{1, \ldots, t\}, S_{2}=\{t+1, \ldots, 2 t\}, S_{3}=\{2 t+1, \ldots, 3 t\}$, and so on. Let $X=A \backslash\left\{x_{1}, \ldots, x_{d}\right\}$. Note that $X$ may be empty. Now construct a profile $P_{N}$ as depicted below:

\footnotetext{
${ }^{4}$ Having already noted $\mathcal{D}_{m}^{*}(n) \subseteq \mathcal{D}_{m}(n)$, we now remark that $\mathcal{D}_{m}^{*}(n)=\{1\} \Longrightarrow \mathcal{D}_{m}(n)=\{1\}$ for all $m, n \in \mathbb{N}$. To prove this by contradiction, we first let $k \in \mathcal{D}_{m}(n) \backslash\{1\}$. Thus, we have $k \in \mathcal{D}(n)$ and $k \leq m$. Due to the fundamental theorem of arithmetic, $k$ has a prime divisor $k^{*}$, which divides $n$ as well, hence $k^{*} \in \mathcal{D}_{m}^{*}(n)$, implying $\mathcal{D}_{m}^{*}(n) \neq\{1\}$.

${ }^{5}$ This condition is equivalent to asking the greatest common divisor of $m$ ! and $n$ to be 1 , as mentioned by Doğan and Giritligil (2015), who reconsider the problem through a group theoretic approach. Interestingly, as Doğan and Giritligil (2015) as well as Bubboloni and Gori (2014) show, $\operatorname{gcd}(m !, n)=1$ turns out to be necessary and sufficient for the existence of anonymous and neutral social welfare functions (i.e., functions which assign to every preference profile a strict ranking of alternatives).

${ }^{6}$ Note that the irresoluteness outlook of an $\operatorname{SCR} f$ does not specify to how many profiles $f$ assigns $k$ alternatives when $k \in K_{f}$.
} 


$$
\begin{array}{ccccc}
S_{1} & S_{2} & S_{3} & \cdots & S_{d} \\
\hline x_{1} & x_{2} & x_{3} & \cdots & x_{d} \\
\cdot & \cdot & \cdot & \cdots & x_{1} \\
\cdot & \cdot & \cdot & \cdots & \cdot \\
\cdot & \cdot & . & \cdots & \cdot \\
. & . & x_{d} & \cdots & x_{d-3} \\
. & x_{d} & x_{1} & \cdots & x_{d-2} \\
x_{d} & x_{1} & x_{2} & \cdots & x_{d-1} \\
X & X & X & \cdots & X
\end{array}
$$

As $f$ is efficient, $f\left(P_{N}\right) \subseteq\left\{x_{1}, \ldots, x_{d}\right\}$. Note that when $d=1$, this implies $\# f\left(P_{N}\right)=1$, hence $1 \in K_{f}$. Now let $d>1$. Consider the permutation $\sigma$ of $A$ such that

$$
\begin{aligned}
\sigma\left(x_{i}\right) & =x_{i+1} \forall i \in\{1, \ldots, d-1\}, \\
\sigma\left(x_{d}\right) & =x_{1}, \text { and } \\
\sigma(\bar{x}) & =\bar{x} \forall \bar{x} \in X .
\end{aligned}
$$

Next, let $\sigma^{\prime} \in \Sigma_{N}$ be such that $\sigma^{\prime}(i)=i-t$ for all $i \in\{t+1, \ldots, d t=n\}$ and $\sigma^{\prime}(i)=(d-1) t+i$ for all $i \in\{1, \ldots, t\}$. Note that $\sigma\left(P_{N}\right)=\left(\sigma^{\prime}\right)^{-1}\left(P_{N}\right)$. Since $f\left(P_{N}\right)$ is a nonempty subset of $A$, there exists $x_{i} \in f\left(P_{N}\right)$ for some $i \in\{1, \ldots, m\}$. By neutrality, $x_{i} \in f\left(P_{N}\right)$ implies $\sigma\left(x_{i}\right) \in f\left(\sigma\left(P_{N}\right)\right)$. By anonymity, $x_{i} \in f\left(P_{N}\right)$ implies $x_{i} \in f\left(\sigma\left(P_{N}\right)\right)$. This is only possible when $f\left(P_{N}\right)=\left\{x_{1}, \ldots, x_{d}\right\}$, as $x_{i} \in f\left(P_{N}\right)$ implies both $x_{i} \in f\left(\sigma\left(P_{N}\right)\right)$ and $x_{i+1} \in f\left(\sigma\left(P_{N}\right)\right)$ for all $i \in\{1, \ldots, d-1\}$ and $x_{d} \in f\left(P_{N}\right)$ implies both $x_{d} \in f\left(\sigma\left(P_{N}\right)\right)$ and $x_{1} \in f\left(\sigma\left(P_{N}\right)\right)$. Hence, $\# f\left(\sigma\left(P_{N}\right)\right)=d$, thus $d \in K_{f}$.

ii. Let $\tau\left(x, P_{N}\right)=\#\left\{i \in N: x P_{i} y \forall y \in A \backslash\{x\}\right\}$ denote the number of individuals that rank $x$ on top of their preferences in the profile $P_{N}$. Define the plurality rule $\Upsilon: \mathcal{L}(A)^{N} \rightarrow \mathcal{A}$ so that

$$
\Upsilon\left(P_{N}\right)=\left\{x \in A: \tau\left(x, P_{N}\right) \geq \tau\left(y, P_{N}\right) \forall y \in A\right\} .
$$

We now define the iterative plurality rule $v: \bigcup_{B \in \mathcal{A}} \mathcal{L}(B)^{N} \rightarrow \mathcal{A}$ such that $v\left(P_{N}\right) \subseteq B$ for all $P_{N} \in \mathcal{L}(B)^{N}$ and $B \in \mathcal{A}$, which selects the plurality winners after successive restriction of profiles to plurality winners. ${ }^{7}$ Let $\Upsilon^{i+1}\left(P_{N}\right)=\Upsilon\left(\left.P_{N}\right|_{\Upsilon^{i}\left(P_{N}\right)}\right)$ for all $i \geq 1$ and $\Upsilon^{1}\left(P_{N}\right)=\Upsilon\left(P_{N}\right)$. Define

$$
v\left(P_{N}\right)=\Upsilon^{k}\left(P_{N}\right),
$$

where $k$ is the minimal integer that satisfies $\Upsilon^{k}\left(P_{N}\right)=\Upsilon^{k+1}\left(P_{N}\right)$. Such an integer always exists given the finiteness of $A$. It is easily checked that $v$ is anonymous, efficient, and neutral. We will show that $K_{v}=\mathcal{D}_{m}(n)$ for all $n, m \in \mathbb{N}$. By definition, for any $x, y \in A$ and any $P_{N} \in \mathcal{L}(A)^{N}, x, y \in v\left(P_{N}\right)$ implies $\tau\left(x,\left.P_{N}\right|_{\Upsilon^{k}\left(P_{N}\right)}\right)=\tau\left(y,\left.P_{N}\right|_{\Upsilon^{k}\left(P_{N}\right)}\right)=t$ for some $t \in \mathbb{N}$. Furthermore, for

\footnotetext{
${ }^{7}$ Note that the iterative plurality rule presents a generalization of our concept of an SCR in the sense that it is defined on the domain of preference profiles over every $B \in \mathcal{A}$.
} 
any $z \notin v\left(P_{N}\right), \tau\left(z,\left.P_{N}\right|_{\Upsilon^{k}\left(P_{N}\right)}\right)=0$. Thus, we have $\left(\# v\left(P_{N}\right)\right) t=n$, implying $K_{v} \subseteq \mathcal{D}(n)$. As $\# v\left(P_{N}\right) \leq m$, in fact, $K_{v} \subseteq \mathcal{D}_{m}(n)$. Given part $\mathrm{i}$ of the theorem, we have $K_{v}=\mathcal{D}_{m}(n)$.

Theorem 2 generalizes Theorem 1, which now comes as a corollary; when Condition $\mu(m, n)$ fails, by Theorem 2.i, every anonymous, efficient, and neutral SCR has $\# f\left(P_{N}\right)>1$ for some $P_{N} \in \mathcal{L}(A)^{N}$ and when Condition $\mu(m, n)$ is satisfied, the iterative plurality rule $v$ ensures the existence of an anonymous, efficient, and neutral $f$ with $\# f\left(P_{N}\right)=1$ for all $P_{N} \in \mathcal{L}(A)^{N}$.

As a matter of fact, Theorem 2 establishes that the irresoluteness outlook $K_{v}$ of the iterative plurality rule is the best that an anonymous, efficient, and neutral SCR can achieve. To be sure, this does not mean that $v$ cannot be refined while preserving anonymity, neutrality, and efficiency. But surely, any anonymous, efficient, and neutral refinement of $v$ will have the same irresoluteness outlook as $v$ itself. Moreover, as we formally state in the next theorem, any anonymous, efficient, and neutral SCR whose irresoluteness outlook is a proper superset of $K_{v}$ can be refined while anonymity, neutrality, and efficiency are preserved. Before, we define, for any $\operatorname{SCR} f: \mathcal{L}(A)^{N} \rightarrow \mathcal{A}$, the composite rule of $: \mathcal{L}(A)^{N} \rightarrow \mathcal{A}$ by vf $\left(P_{N}\right)=v\left(\left.P_{N}\right|_{f\left(P_{N}\right)}\right) \forall P_{N} \in \mathcal{L}(A)^{N}$.

Theorem 3 Given a social choice problem $(A, N)$, an anonymous, efficient, and neutral $S C R f$ with $K_{f} \supset K_{v}$ admits the anonymous, efficient, and neutral refinement of $: \mathcal{L}(A)^{N} \rightarrow \mathcal{A}$ with $K_{\text {vf }}=K_{v}$.

Proof Take any social choice problem $(A, N)$ and any anonymous, efficient, and neutral $f$ with $K_{f} \supset K_{v}$. By definition of $v f$, we have $v f\left(P_{N}\right) \subseteq f\left(P_{N}\right)$ for all $P_{N} \in \mathcal{L}(A)^{N}$. Take any $P_{N}^{\prime} \in \mathcal{L}(A)^{N}$. First, let $\# f\left(P_{N}^{\prime}\right) \in K_{v}$. As $K_{v}=\mathcal{D}_{m}(n)$ by Theorem 2 , we have $\# f\left(P_{N}^{\prime}\right) \in \mathcal{D}_{m}(n)$ which implies $\# v f\left(P_{N}^{\prime}\right) \in K_{v}$ as well. Now, let \#f(P $\left.P_{N}^{\prime}\right) \in K_{f} \backslash K_{v}$. As $\# v f\left(P_{N}^{\prime}\right) \in \mathcal{D}(n)$ by definition of $v$, we cannot have $v f\left(P_{N}^{\prime}\right)=f\left(P_{N}^{\prime}\right)$. Thus, of $\left(P_{N}^{\prime}\right) \subset f\left(P_{N}^{\prime}\right)$. Furthermore, as $\# v f\left(P_{N}^{\prime}\right) \leq m$, we have $\# v f\left(P_{N}^{\prime}\right) \in \mathcal{D}_{m}(n)=K_{v}$. Noting that $v f$ is anonymous, efficient, and neutral concludes the proof.

As an instance of Theorem 3, consider the social choice problem $A=\{x, y, z\}$, $N=\{1,2\}$, and $P_{N} \in \mathcal{L}(A)^{N}$ with $x P_{1} y P_{1} z$ and $z P_{2} y P_{2} x$. For the Borda rule $\beta$, which chooses the alternatives that have the minimal sum of ranks over individuals, we have $\beta\left(P_{N}\right)=\{x, y, z\}$, thus $3 \in K_{\beta}$, while one can check that $K_{v}=\{1,2\}$, and, indeed, the composite rule $v \beta$, which gives $v \beta\left(P_{N}\right)=\{x, z\} \subset \beta\left(P_{N}\right)$, refines $\beta$ and is anonymous, efficient, and neutral.

Theorem 3 points to the possibility of shrinking the irresoluteness outlook of an anonymous, efficient, and neutral SCR $f$ down to $K_{v}$ by composing $f$ with $v$, while of preserves all three properties. However, this does not mean that of can refine $f$ at

\footnotetext{
${ }^{8}$ Part (ii) of Theorem 2 can be proven with rules other than the iterative plurality rule, such as Coombs rule, as in Moulin (1983) p. 24. We are thankful to a referee for pointing out this fact.
} 
every $P_{N} \in \mathcal{L}(A)^{N}$ with $f\left(P_{N}\right) \supset v\left(P_{N}\right)$. To see this, let $m=5$ and $n=6$, and consider the following profile $P_{N}$.

\begin{tabular}{cccccc}
$P_{1}$ & $P_{2}$ & $P_{3}$ & $P_{4}$ & $P_{5}$ & $P_{6}$ \\
\hline$x$ & $x$ & $t$ & $r$ & $y$ & $y$ \\
$y$ & $y$ & $z$ & $z$ & $x$ & $x$ \\
$z$ & $z$ & $x$ & $y$ & $r$ & $z$ \\
$r$ & $t$ & $y$ & $x$ & $z$ & $t$ \\
$t$ & $r$ & $r$ & $t$ & $t$ & $r$
\end{tabular}

We have $v\left(P_{N}\right)=\{x, y\}$ and $\varphi\left(P_{N}\right)=\{x, y, z\}$, where $\varphi$ denotes the fallback bargaining rule. ${ }^{9}$ However, the composite rule $v \varphi$ does not refine $\varphi$ at this profile, i.e., $v \varphi\left(P_{N}\right)=\{x, y, z\}$.

\section{Consequential neutrality}

Let $W_{f}(S)=\left\{P_{N} \in \mathcal{L}(A)^{N}: f\left(P_{N}\right)=S\right\}$ for any $S \in \mathcal{A}$ be the set of profiles to which an SCR $f$ assigns $S$. We write $W_{f}(x)$ in place of $W_{f}(\{x\})$ in case of singletons. Define an SCR $f: \mathcal{L}(A)^{N} \rightarrow \mathcal{A}$ to be consequentially neutral $(\mathrm{CN})$ iff $\# W_{f}(S)=\# W_{f}\left(S^{\prime}\right)$ for all $S, S^{\prime} \in \mathcal{A}$ with $\# S=\# S^{\prime}$. Thus, when $f$ is resolute, $\mathrm{CN}$ requires that any two alternatives are assigned to the same number of profiles.

Proposition 1 An SCR $f$ is neutral only iff is $C N$.

Proof Take any neutral SCR $f$. Let $S, S^{\prime} \subseteq A$ be such that $S=\left\{x_{1}, \ldots, x_{h}\right\}$ and $S^{\prime}=\left\{x_{1}^{\prime}, \ldots, x_{h}^{\prime}\right\}$ for some $h \in\{1, \ldots, m\}$. Take any $P_{N} \in W_{f}(S)$. Thus, we have $f\left(P_{N}\right)=S$. Take any $\sigma: A \leftrightarrow A$ such that $\sigma\left(x_{i}\right)=x_{i}^{\prime}$ for all $i \in\{1, \ldots, h\}$. By neutrality, $P_{N} \in W_{f}(S)$ implies $f\left(\sigma\left(P_{N}\right)\right)=S^{\prime}$, or $\sigma\left(P_{N}\right) \in W_{f}\left(S^{\prime}\right)$. Thus, we have $\# W_{f}(S) \leq \# W_{f}\left(S^{\prime}\right)$. As \#政 $\left(S^{\prime}\right) \leq \# W_{f}(S)$ holds as well, the proof is completed.

Now, we focus on resolute SCRs. Given $m, n \in \mathbb{N} \backslash\{1\}$ with $\# A=m$ and $N=\{1, \ldots, n\}$, we write $\mathcal{F}_{m, n}^{C N}$ for the set of resolute SCRs that are $\mathrm{CN} ; \mathcal{F}_{m, n}^{\text {NEUTRAL }}$ for the set of resolute and neutral SCRs; and $\mathcal{F}^{\text {RESOLUTE }}$ for the set of resolute SCRs.

We know from Proposition 1 that $\mathcal{F}_{m, n}^{N E U T R A L} \subseteq \mathcal{F}_{m, n}^{C N}$ for all $m, n \in \mathbb{N} \backslash\{1\}$. The inclusion is indeed strict. To see this, fix a profile $P_{N} \in \mathcal{L}(A)^{N}$. Take the permutation $\sigma \in \Sigma_{A}$ such that $\sigma\left(x_{1}\right)=x_{2}, \sigma\left(x_{2}\right)=x_{1}$, and if $m \geq 3$, let $\sigma\left(x_{j}\right)=x_{j}$ for all $j \in\{3, \ldots, m\}$. Take any partition $\mathcal{P}=\left\{\mathcal{P}_{1}, \ldots, \mathcal{P}_{m}\right\}$ of $\mathcal{L}(A)^{N}$ such that $\# \mathcal{P}_{i}=\# \mathcal{P}_{j}$ for all $i, j \in\{1, \ldots, m\}$ and $\bar{P}_{N}, \sigma\left(\bar{P}_{N}\right) \in \mathcal{P}_{1}$. Define $f: \mathcal{L}(A)^{N} \rightarrow A$

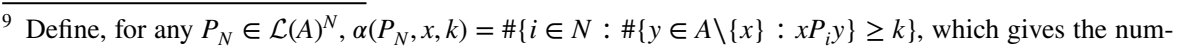
ber of individuals that rank $x$ higher than at least $k$ alternatives. Now, define the fallback bargaining rule $\varphi: \mathcal{L}(A)^{N} \rightarrow \mathcal{A}$ so that, $\forall x \in A, x \in \varphi\left(P_{N}\right)$ iff

$$
{ }^{*} \max _{k \in\{0, \ldots, m-1\}}\left\{k \in \mathbb{N}: \alpha\left(P_{N}, x, k\right)=n\right\} \geq * \max _{k \in\{0, \ldots, m-1\}}\left\{k \in \mathbb{N}: \alpha\left(P_{N}, y, k\right)=n\right\}
$$

for all $y \in A \backslash\{x\}$.
} 
such that $f\left(P_{N}\right)=x_{i}$ for all $P_{N} \in \mathcal{P}_{i}$ and $i \in\{1, \ldots, m\}$. By construction, $\#\left\{P_{N} \in \mathcal{L}(A)^{N}: f\left(P_{N}\right)=x_{i}\right\}=\#\left\{P_{N} \in \mathcal{L}(A)^{N}: f\left(P_{N}\right)=x_{j}\right\}$ for all $i, j \in\{1, \ldots, m\}$, hence $f$ is $\mathrm{CN}$. However, it fails neutrality as $f\left(\bar{P}_{N}\right)=x_{1}=f\left(\sigma\left(\bar{P}_{N}\right)\right)$.

This raises the following two issues: How large is $\mathcal{F}_{m, n}^{C N}$ compared to $\mathcal{F}_{m, n}^{N E U T R A L}$ and which interesting resolute SCRs, if any, does it contain? We address the first question through a counting approach. ${ }^{10}$

Theorem 4 The following equalities hold.

i. $\quad \# \mathcal{F}_{m, n}^{\text {RESOLUTE }}=m^{\left(m !^{n}\right)}$.

ii. $\quad \# \mathcal{F}_{m, n}^{N E U T R A L}=m^{\left(m !^{n-1}\right)}$.

iii. $\quad \# \mathcal{F}_{m, n}^{C N}=\frac{\left(m !^{n}\right) ! m !}{\left(m !^{n-1}(m-1) !\right) !^{m}}$.

Proof i. This is straightforward, as we noted before that $\# \mathcal{L}(A)^{N}=m !^{n}$.

ii. We say that $P_{N}^{\prime}$ is a renaming (for alternatives) of $P_{N}$ iff there exists $\sigma \in \Sigma_{A}$ such that $P_{N}^{\prime}=\sigma\left(P_{N}\right)$. We write $P_{N} \rho P_{N}^{\prime}$ when $P_{N}^{\prime}$ is a renaming of $P_{N}$. Noting that $\rho \subseteq \mathcal{L}(A)^{N} \times \mathcal{L}(A)^{N}$ is an equivalence relation, we write $\mathcal{E}$ for the partition of $\mathcal{L}(A)^{N}$ provided by $\rho$. Note that each profile $P_{N}$ admits $m$ ! renamings and that $\# \mathcal{L}(A)^{N}=m !^{n}$. Thus, $\mathcal{E}$ admits $m !^{n-1}$ equivalence classes, each of which contains $m$ ! profiles. We write $\mathcal{E}=\left\{\mathcal{E}_{i}\right\}_{i \in\left\{1, \ldots, m !^{n-1}\right\}}$ with $\# \mathcal{E}_{i}=m$ ! for all $i \in\left\{1, \ldots, m !^{n-1}\right\}$. Take any $\mathcal{E}_{t} \in \mathcal{E}$ and pick any $P_{N} \in \mathcal{E}_{t}$. Let $f\left(P_{N}\right)=x$ for some $x \in A$. Neutrality, together with the definition of $\mathcal{E}_{t}$, determines $f\left(P_{N}^{\prime}\right)$ for all $P_{N}^{\prime} \in \mathcal{E}_{t} \backslash\left\{P_{N}\right\}$. As there are $m$ alternatives that can be assigned to $P_{N}$ by $f$, there are $m$ neutral and resolute ways an SCR can be defined for the profiles in $\mathcal{E}_{t}$. As $t \in\left\{1, \ldots, m !^{n-1}\right\}$, there are $m^{\left(m !^{n-1}\right)}$ neutral and resolute SCRs altogether.

iii. $\quad$ First observe that, given any two natural numbers $p$ and $q$, there are $\frac{(p q) !}{q ! p}$ ways to partition a set of cardinality $p q$ into $p$ sets, each with cardinality $q$. Hence, there are $\frac{\left(m !^{n}\right) !}{\left(m !^{n-1}(m-1) !\right) !^{m}}$ ways to partition $\mathcal{L}(A)^{N}$ with cardinality $m !^{n}$ into $m$ sets, each with cardinality $m !^{n} / m=m !^{n-1}(m-1) !$. For each of these ways, $m$ ! distinct resolute SCRs can be defined. As a result, $\frac{\left(m !^{n}\right) !}{\left(m !^{n-1}(m-1) !\right) ! m} m !$ resolute SCRs that satisfy consequential neutrality can be constructed.

From Theorem 4, one can compute $\frac{\# \mathcal{F}_{m, n}^{\text {NEUTRAL }}}{\# \mathcal{F}_{m, n}^{\text {RESOLUTE }}}=m^{\left(-1+\frac{1}{m !}\right) m !^{n}}$. As $\frac{1}{m !}<1$ for all $m>1$, this ratio approaches to 0 as $m \rightarrow \infty$ or $n \rightarrow \infty$. Thus, we conclude that the

\footnotetext{
10 Perry and Powers (2008) propose a method to count, in the case of two alternatives, the number of aggregation rules that satisfy anonymity and neutrality. Bubboloni and Gori (2016) give a method to perform these counts for any number of alternatives.
} 
ratio of the number of neutral and resolute SCRs to the number of all resolute SCRs is negligible in the limit. Although we do not have analytical solutions for the comparisons regarding consequential neutrality, we obtained some numerical observations through computations for small values of $m$ and $n$ that are provided in Appendix A. These indicate that both $\frac{\# \mathcal{F}_{m, n}^{C N}}{\# \mathcal{F}_{m, n}^{\text {RESOLUTE }}}$ and $\frac{\# \mathcal{F}_{m, n}^{\text {NEUTRAL }}}{\# \mathcal{F}_{m, n}^{C N}}$ converge to 0 , as $m$ or $n$ increases. Thus, although consequential neutrality and neutrality are both hard to satisfy, neutrality seems to be considerably more demanding compared to consequential neutrality. ${ }^{11}$

Now we address whether $\mathcal{F}_{m, n}^{C N} \backslash \mathcal{F}_{m, n}^{\text {NEUTRAL }}$ contains interesting SCRs and the answer is affirmative, at least for certain sizes of the social choice problem.

We say that $(m, n)$ satisfies Condition $\gamma(m, n)$ iff $m \mid\left(\begin{array}{c}m \\ k\end{array}\right)$ for all $k \in \mathcal{D}_{m}(n)$. This condition ensures the existence of anonymous, $\mathrm{CN}$, efficient, and resolute SCRs, as shown in Theorem 5 below.

Theorem 5 Let $(A, N)$ be a social choice problem with size $(m, n)$ where Condition $\gamma(m, n)$ is satisfied. Every anonymous, efficient, irresolute, and neutral SCR f admits a resolute refinement which is anonymous, $C N$, and efficient.

Proof Fix $m, n \in \mathbb{N}$ as such, let $A=\left\{x_{1}, \ldots, x_{m}\right\}$, and take any anonymous, efficient, and neutral $f: \mathcal{L}(A)^{N} \rightarrow \mathcal{A}$. Clearly, $\# v f\left(P_{N}\right) \in \mathcal{D}_{m}(n)$ for all $P_{N} \in \mathcal{L}(A)^{N}$ due to Theorems 2 and 3. Next, observe that $m \nmid n$, as otherwise we would have $m \in \mathcal{D}_{m}(n)$, while $m+\left(\begin{array}{l}m \\ m\end{array}\right)=1$. Thus, there does not exist a profile $P_{N}$ with $v f\left(P_{N}\right)=A$. For any $k \in \mathcal{D}_{m}(n) \backslash\{1\}$, denote with $\mathcal{A}_{k}$ the subsets of $A$ with precisely $k$ elements, that is, $\mathcal{A}_{k}=\{S \subseteq A: \# S=k\}$. Thus, we have \# $\mathcal{A}_{k}=\left(\begin{array}{c}m \\ k\end{array}\right)=m\left(\left(\begin{array}{c}m-1 \\ k-1\end{array}\right) / k\right)$. Condition $\gamma(m, n)$ ensures that $\left(\begin{array}{c}m-1 \\ k-1\end{array}\right) / k(=t$ from now on $)$ is a natural number. We want to show that each $x_{i} \in A$ can be assigned to $t$ distinct sets of cardinality $k$ that contains $x_{i}$. This will suffice to prove the theorem, given Proposition 1, as the number of profiles these $k$-sets are assigned to under a neutral SCR are equal. To do that, we introduce the following iterative approach.

First, define $\sigma: A \leftrightarrow A$ such that $\sigma\left(x_{i}\right)=x_{i+1}$ for all $i \in\{1, \ldots, m-1\}$ and $\sigma\left(x_{m}\right)=x_{1}$. Next, let $>_{\mathcal{A}_{k}}$ denote the lexicographic order of the $k$-sets in $\mathcal{A}_{k}$ according to the order $x_{1}>\cdots>x_{m}$. Thus, for instance, $\left\{x_{1}, \ldots, x_{k}\right\}>_{\mathcal{A}_{k}}\left\{x_{1}, \ldots, x_{k-1}, x_{k+1}\right\}>_{\mathcal{A}_{k}} S$ for all $S \in \mathcal{A}_{k} \backslash\left\{\left\{x_{1}, \ldots, x_{k}\right\},\left\{x_{1}, \ldots, x_{k-1}, x_{k+1}\right\}\right\}$.

\footnotetext{
${ }_{11}$ We are providing computational results for only some small values of $m$ and $n$ because as $m$ and $n$ increase, these values grow dramatically (see Tables 2 and 3 in the Appendix). As diminution in the ratios are also fast, these values appear to be sufficient for this conclusion. Since the aim of this counting exercise is to assess the comparison of numbers of functions that satisfy the two versions of neutrality, we leave out other axioms (such as anonymity and efficiency), although it certainly is an interesting question.
} 
Step 1.

- Take the first set in $\mathcal{A}_{k}$ according to $>_{\mathcal{A}_{k}}$, i.e., $\left\{x_{1}, \ldots, x_{k}\right\}$, and denote this set with $A_{k, 1}$. Now, denote with $\sigma^{r}\left(A_{k, 1}\right)$, the $r$-time application of permutation $\sigma$, with a slight abuse of notation. Thus, for instance, $\sigma^{1}\left(A_{k, 1}\right)=\left\{x_{2}, \ldots, x_{k+1}\right\}$. Denote with $\mathcal{A}_{k, 1}$ the set comprising of $A_{k, 1}$ together with $m-1$ other $k$-sets in $\mathcal{A}_{k}$ that are constructed from $A_{k, 1}$ by applying $\sigma$ iteratively $m-1$ times. Thus, $\mathcal{A}_{k, 1}=\left\{A_{k, 1}, \sigma^{1}\left(A_{k, 1}\right), \ldots, \sigma^{m-1}\left(A_{k, 1}\right)\right\}$.

\section{Step 2.}

- Take the first set in $\mathcal{A}_{k} \backslash \mathcal{A}_{k, 1}$ according to $>_{\mathcal{A}_{k}}$ and denote this set with $A_{k, 2}$. Construct $\mathcal{A}_{k, 2}$ as in Step 1 so that $\mathcal{A}_{k, 2}=\left\{A_{k, 2}, \sigma^{1}\left(A_{k, 2}\right), \ldots, \sigma^{m-1}\left(A_{k, 2}\right)\right\}$.

Step $j$ for $j \in\{3, \ldots, t\}$ if $t>2$.

- Take the first set in $\mathcal{A}_{k} \backslash \bigcup_{c=1}^{j-1} \mathcal{A}_{k, c}$ according to $>_{\mathcal{A}_{k}}$ and denote this set with $A_{k, j}$. Construct $\mathcal{A}_{k, j}$ as in Step 1 .

The proof concludes by constructing an SCR $g$ that refines of such that for all $P_{N} \in \mathcal{L}(A)^{N}, k \in \mathcal{D}_{m}(n) \backslash\{1\}$, and $j \in\{1, \ldots, t\}$,

- $v f\left(P_{N}\right)=A_{k, j} \Longrightarrow g\left(P_{N}\right)=x_{1}$ and

- $v f\left(P_{N}\right)=\sigma^{r}\left(A_{k, j}\right) \Longrightarrow g\left(P_{N}\right)=x_{r+1} \forall r \in\{1, \ldots, m-1\}$.

We need to make sure that the following hold.

(i) $x_{1} \in A_{k, j}$ for all $j \in\{1, \ldots, t\}$.

(ii) $x_{r+1} \in \sigma^{r}\left(A_{k, j}\right)$ for all $j \in\{1, \ldots, t\}$ and for all $r \in\{1, \ldots, m-1\}$.

(iii) $\left\{\mathcal{A}_{k, j}\right\}_{j \in\{1, \ldots, t\}}$ is a partition of $\mathcal{A}_{k}$.

(i) First, note that $x_{1} \in A_{k, 1}$. Next, observe that $\#\left\{S \in \mathcal{A}_{k, 1}: x_{1} \in S\right\}=k$ as $\# A_{k, 1}=k, x_{1} \in A_{k, 1}$, and for each $x_{j} \in A_{k, 1} \backslash\left\{x_{1}\right\}$, there exists a unique $r \in\{1, \ldots, m-1\}$ such that $\sigma^{r}\left(x_{j}\right)=x_{1}$. Thus, in fact, for all $j \in\{2, \ldots, t\}$, $\bigcup_{c=1}^{j-1} \mathcal{A}_{k, c}$ contains $(j-1) k$ sets that contain $x_{1}$ and there remain $(t-j+1) k$ sets in $\mathcal{A}_{k} \backslash \bigcup_{j=1}^{j-1} \mathcal{A}_{k, c}$ that contain $x_{1}{ }^{12}$ As for each $j \in\{2, \ldots, t\}$, the first set in $\mathcal{A}_{k} \backslash \bigcup_{c=1}^{j} \mathcal{A}_{k, c}$ according to $>_{\mathcal{A}_{k}}$ is chosen to be $A_{k, j}, x_{1} \in A_{k, j}$ for all $j \in\{1, \ldots, t\}$ is established.

(ii) As $x_{1} \in A_{k, j}$ for all $j \in\{1, \ldots, t\}$ and $\sigma^{r}\left(x_{1}\right)=x_{r+1}$, it is straightforward to note that $x_{r+1} \in \sigma^{r}\left(A_{k, j}\right)$ for all $j \in\{1, \ldots, t\}$ and for all $r \in\{1, \ldots, m-1\}$.

12 This holds for any $x_{i} \in A$. 
Table 1 Iterative process for constructing the partitioning $\left\{\mathcal{A}_{5, j}\right\}_{j \in\{1, \ldots, t\}}$ of $\mathcal{A}_{5}$, where $t=\left(\begin{array}{l}7 \\ 5\end{array}\right) / 7=3$

\begin{tabular}{llll}
\hline & $j=1$ & $j=2$ & $j=3$ \\
\hline$A_{5, j}$ & $\left\{x_{1}, x_{2}, x_{3}, x_{4}, x_{5}\right\}$ & $\left\{x_{1}, x_{2}, x_{3}, x_{4}, x_{6}\right\}$ & $\left\{x_{1}, x_{2}, x_{3}, x_{5}, x_{6}\right\}$ \\
$\sigma\left(A_{5, j}\right)$ & $\left\{x_{2}, x_{3}, x_{4}, x_{5}, x_{6}\right\}$ & $\left\{x_{2}, x_{3}, x_{4}, x_{5}, x_{7}\right\}$ & $\left\{x_{2}, x_{3}, x_{4}, x_{6}, x_{7}\right\}$ \\
$\sigma^{2}\left(A_{5, j}\right)$ & $\left\{x_{3}, x_{4}, x_{5}, x_{6}, x_{7}\right\}$ & $\left\{x_{3}, x_{4}, x_{5}, x_{6}, x_{1}\right\}$ & $\left\{x_{3}, x_{4}, x_{5}, x_{7}, x_{1}\right\}$ \\
$\sigma^{3}\left(A_{5, j}\right)$ & $\left\{x_{4}, x_{5}, x_{6}, x_{7}, x_{1}\right\}$ & $\left\{x_{4}, x_{5}, x_{6}, x_{7}, x_{2}\right\}$ & $\left\{x_{4}, x_{5}, x_{6}, x_{1}, x_{2}\right\}$ \\
$\sigma^{4}\left(A_{5, j}\right)$ & $\left\{x_{5}, x_{6}, x_{7}, x_{1}, x_{2}\right\}$ & $\left\{x_{5}, x_{6}, x_{7}, x_{1}, x_{3}\right\}$ & $\left\{x_{5}, x_{6}, x_{7}, x_{2}, x_{3}\right\}$ \\
$\sigma^{5}\left(A_{5, j}\right)$ & $\left\{x_{6}, x_{7}, x_{1}, x_{2}, x_{3}\right\}$ & $\left\{x_{6}, x_{7}, x_{1}, x_{2}, x_{4}\right\}$ & $\left\{x_{6}, x_{7}, x_{1}, x_{3}, x_{4}\right\}$ \\
$\sigma^{6}\left(A_{5, j}\right)$ & $\left\{x_{7}, x_{1}, x_{2}, x_{3}, x_{4}\right\}$ & $\left\{x_{7}, x_{1}, x_{2}, x_{3}, x_{5}\right\}$ & $\left\{x_{7}, x_{1}, x_{2}, x_{4}, x_{5}\right\}$ \\
& $\mathcal{A}_{5,1}$ & $\mathcal{A}_{5,2}$ & $\mathcal{A}_{5,3}$ \\
\hline & & & \\
\hline
\end{tabular}

(iii) First, note that $\# \mathcal{A}_{k, i}=m$ for all $i \in\{1, \ldots, t\}$. We need to show that $\mathcal{A}_{k, i} \cap \mathcal{A}_{k, j}=\emptyset$ for any $i, j \in\{1, \ldots, t\}$. This follows from the statement $(i)$ as $A_{k, j} \in \mathcal{A}_{k} \backslash \bigcup_{c=1}^{j-1} \mathcal{A}_{k, c}$ for all $j \in\{1, \ldots, t\}$. To see that, suppose for a contradiction that there exists $S \in \mathcal{A}_{k, i} \cap \mathcal{A}_{k, j}$ for some $i, j \in\{1, \ldots, t\}$ with $j>i$. We must have $S=\sigma^{r_{i}}\left(A_{k, i}\right)=\sigma^{r_{j}}\left(A_{k, j}\right)$ for some $r_{i}, r_{j} \in\{1, \ldots, m-1\}$. Note that $r_{i} \neq r_{j}$ as $A_{k, i} \neq A_{k, j}$. In case $r_{i}>r_{j}$, we have $\sigma^{r_{i}-r_{j}}\left(A_{k, i}\right)=A_{k, j}$ and in case $r_{i}<r_{j}$, we have $\sigma^{r_{i}+m-r_{j}}\left(A_{k, i}\right)=A_{k, j}$, both contradicting that $A_{k, j} \in \mathcal{A}_{k} \backslash \bigcup_{c=1}^{j-1} \mathcal{A}_{k, c}{ }^{13}$

We let $g\left(P_{N}\right)=v f\left(P_{N}\right)$ for all $P_{N} \in \mathcal{L}(A)^{N}$ with $\# v f\left(P_{N}\right)=1$. $g$ is a resolute refinement of $f$. Naturally, $g$ is anonymous as $f$ is. Furthermore, by construction of the iterative process above, $g$ is also CN. Finally, as $f$ is efficient, $g$ is efficient as well.

To see how the refinement in Theorem 5 can be constructed, consider the following example with $A=\left\{x_{1}, \ldots, x_{7}\right\}$ and $n=5$. Take any $f$ that is anonymous, efficient, and neutral. We have $\mathcal{D}_{7}(5)=\{1,5\}$, so we focus on $k=5$ for the iterative process. We have $\left(\begin{array}{l}7 \\ 5\end{array}\right)=21$ and $t=3$, that is, there are 21 distinct sets with 5 alternatives and all profiles that result in 5 alternatives under of should be assigned to each of the 7 alternatives so that each will be chosen within 3 different sets. So in Step 1, we set $A_{5,1}=\left\{x_{1}, x_{2}, x_{3}, x_{4}, x_{5}\right\}$ and construct $\mathcal{A}_{5,1}$. In Step 2, we set $A_{5,2}=\left\{x_{1}, x_{2}, x_{3}, x_{4}, x_{6}\right\}$ which follows $\left\{x_{1}, x_{2}, x_{3}, x_{4}, x_{5}\right\}$ in the lexicographic order $>_{\mathcal{A}_{5}}$ and construct $\mathcal{A}_{5,2}$. In Step 3, final step, we cannot set the next set according to $>_{\mathcal{A}_{5}}$, i.e., $\left\{x_{1}, x_{2}, x_{3}, x_{4}, x_{7}\right\}$, as $A_{5,3}$ because it already appears in $\mathcal{A}_{5,1}$ as $\sigma^{6}\left(A_{5,1}\right)=\left\{x_{1}, x_{2}, x_{3}, x_{4}, x_{7}\right\}$. However, we can set $\left\{x_{1}, x_{2}, x_{3}, x_{5}, x_{6}\right\}$ as $A_{5,3}$, which follows $\left\{x_{1}, x_{2}, x_{3}, x_{4}, x_{7}\right\}$ in the lexicographic order $>_{\mathcal{A}_{5}}$ and construct $\mathcal{A}_{5,3}$ to complete the iterative process as depicted in the Table 1.

The refinement $g$ is constructed so that for all $P_{N} \in \mathcal{L}(A)^{N}$ and for all $j \in\{1,2,3\}$

(i) of $\left(P_{N}\right) \in A_{5, j}$ implies $g\left(P_{N}\right)=x_{1}$ and

(ii) $v f\left(P_{N}\right) \in \sigma^{r}\left(A_{5, j}\right)$ implies $g\left(P_{N}\right)=x_{r+1}$ for all $r \in\{1, \ldots, 6\}$.

13 Note that $\sigma^{r+m}\left(x_{i}\right)=\sigma^{r}\left(x_{i}\right)$ for all $r \in \mathbb{N}$ and $i \in\{1, \ldots, m\}$. 
Note that Condition $\gamma(m, n)$ ensures the existence of anonymous, $\mathrm{CN}$, efficient, and resolute SCRs by asking $m \mid\left(\begin{array}{c}m \\ k\end{array}\right)$ to hold for every $k \in \mathcal{D}_{m}(n)$. On the other hand, as it follows from the proof of Theorem 5, a weaker version of Condition $\gamma(m, n)$ that asks $m \mid\left(\begin{array}{c}m \\ k\end{array}\right)$ to hold for some $k \in \mathcal{D}_{m}(n)$, although does not ensure resoluteness, allows the existence of anonymous and efficient refinements that satisfy $\mathrm{CN}$. We state this formally in the following remark and leave out its proof as it follows from the proof of Theorem 5. Let us say that $(m, n)$ satisfies Condition $\gamma^{\prime}(m, n)$ iff $m \mid\left(\begin{array}{c}m \\ k\end{array}\right)$ for some $k \in \mathcal{D}_{m}(n)$.

Remark 1 Let $(A, N)$ be a social choice problem with size $(m, n)$ where Condition $\gamma^{\prime}(m, n)$ is satisfied. Every anonymous, efficient, and neutral SCR $f$ admits a refinement which is anonymous, efficient, and $C N$.

How restrictive is Condition $\gamma(m, n)$ ? Note that when $m$ is a prime, Condition $\gamma(m, n)$ is satisfied. ${ }^{14}$ Thus, anonymous, CN, efficient, and resolute SCRs exist when $m$ is prime and does not divide $n$. Campbell and Kelly (2015) show that when $n$ has at least two distinct prime factors, there can only be finitely many values of $m$ for which there are anonymous, neutral, and resolute SCRs. Our result implies, for instance, that for such $n$, there are infinitely many values of $m$ (such as all primes that are greater than $n$ ) for which there are anonymous, CN, and resolute SCRs. Furthermore, these SCRs can be efficient.

To expand the picture drawn by Theorem 5 , we show that Condition $\gamma(m, n)$ is not necessary for the existence of anonymous, CN, efficient, and resolute SCRs. As a matter of fact, the theorem below spans some instances where Condition $\gamma(m, n)$ fails, e.g., $m=4$ and $n=2$ (note that $2 \in \mathcal{D}_{4}(2)$ and $4+\left(\begin{array}{l}4 \\ 2\end{array}\right)$ ).

Theorem 6 Any social choice problem $(A, N)$ with $m \geq 4$ and $n \in\{2,3\}$ admits an anonymous, CN, efficient, and resolute $S C R$.

Proof Let $m \geq 4$ and $N=\{1,2\}$. For any $x, y \in A$, let $T_{x y} \subset \mathcal{L}(A)^{N}$ denote the set of profiles where individual 1 ranks $x$ first and individual 2 ranks $y$ first. Hence $\left\{T_{x y}\right\}_{x \neq y}$ partitions the set of profiles where there is no unanimously top ranked alternative. Given any $x, y \in A$, note that

$$
T_{y x}=\left\{(Q, P) \in \mathcal{L} \Leftarrow \mathcal{A} \Rightarrow^{N}:(P, Q) \in T_{x y}\right\},
$$

and hence, $\# T_{x y}=\# T_{y x}$. Now, let $A=\left\{x_{1}, \ldots, x_{m}\right\}$ and define for any distinct $i, j \in\{1, \ldots, m\}$,

$$
D_{m}(i, j)=\{k, l \in\{1, \ldots, m\} \backslash\{i, j\}: k<l<t \forall t \in\{1, \ldots, m\} \backslash\{i, j, k, l\}\},
$$

\footnotetext{
${ }^{14}$ To see this, note that the numerator in $\left(\begin{array}{c}m \\ k\end{array}\right)=\frac{m \cdot \ldots \cdot(m-k+1)}{k !}$ is divisible by $m$ whereas none of $\{2, \ldots, k\}$ divides $m$.
} 
as the doubleton that contains the lowest two indices in $A$ excluding $i$ and $j$. Let $g: \mathcal{L}(A)^{N} \rightarrow A$ be a resolute SCR that assigns to any profile the alternative that is ranked first by both individuals, if exists. Note that $\#\left\{P_{N} \in \mathcal{L}(A)^{N}: x P_{i} z \forall z \in A \forall i \in N\right\}=\#\left\{P_{N} \in \mathcal{L}(A)^{N}: y P_{i} z \forall z \in A \forall i \in N\right\}$ for all $x, y \in A$. Furthermore, for all $P_{N} \in T_{x_{i} x_{j}}$ with $i<j$ and $D_{m}(i, j)=\{k, l\}$, let $g\left(P_{N}\right)=x_{i}$ when $x_{k} P_{1} x_{l} \Longleftrightarrow x_{k} P_{2} x_{l}$ and $g\left(P_{N}\right)=x_{j}$ otherwise. Hence, we have $\#\left\{P_{N} \in T_{x_{i} x_{j}}: g\left(P_{N}\right)=x_{i}\right\}=\#\left\{P_{N} \in T_{x_{i} x_{j}}: g\left(P_{N}\right)=x_{j}\right\}$ for all $x_{i}, x_{j} \in A$ such that $i<j$. Furthermore, let $g(P, Q)=g(Q, P) \forall(P, Q) \in \mathcal{L}(A)^{N}$. By construction, $g$ is $\mathrm{CN}$ and anonymous. Moreover, as $g$ picks an alternative only if it is ranked first by an individual, it is also efficient.

Now let $N=\{1,2,3\}$, hence $n=3$. Let $\mathcal{T} \subset \mathcal{L}(A)^{N}$ denote the subset of profiles where each individual has a distinct alternative as most preferred. That is, $P_{N} \in \mathcal{T}$ iff $x_{1} P_{1} y$ for all $y \in A \backslash\left\{x_{1}\right\}, x_{2} P_{2} y$ for all $y \in A \backslash\left\{x_{2}\right\}$, and $x_{3} P_{3} y$ for all $y \in A \backslash\left\{x_{3}\right\}$ implies $x_{1}, x_{2}$, and $x_{3}$ are all distinct. Note that under the plurality rule $\Upsilon$, \# $\Upsilon\left(P_{N}\right)=1$ for all $P_{N} \in \mathcal{L}(A)^{N} \backslash \mathcal{T}$ and $\# \Upsilon\left(P_{N}\right)=3$ for all $P_{N} \in \mathcal{T}$.

Let $A=\left\{x_{1}, \ldots, x_{m}\right\}$. For any $q, r, t \in\{1, \ldots, m\}$ with $q<r<t$, let $M(\{q, r, t\})$ denote the minimal element in $\{1, \ldots, m\} \backslash\{q, r, t\}$. So for instance, when $m=5$, $M(\{2,3,4\})=1$ and $M(\{1,2,4\})=3$. Let $\mathcal{T}_{\left\{x_{q}, x_{r}, x_{t}\right\}} \subset \mathcal{T}$ denote the set of profiles where each of $x_{q}, x_{r}$, and $x_{t}$ appears on top. We now define an SCR $h$. We let $h\left(P_{N}\right)=\Upsilon\left(P_{N}\right)$ for all $P_{N} \in \mathcal{L}(A)^{N} \backslash \mathcal{T}$. For any $P_{N} \in \mathcal{T}_{\left\{x_{q}, x_{r}, x_{t}\right\}}$, let $j \in N$ denote the individual such that $x_{q} P_{j} x_{w}$ for all $w \in\{1, \ldots, m\} \backslash\{q\}$. We let $h\left(P_{N}\right)=x_{q}$ if $x_{M(\{q, r, t\})} P_{j} x_{z}$ for all $z \in\{r, t\}, h\left(P_{N}\right)=x_{r}$ if $x_{z} P_{j} x_{M(\{q, r, t\})}$ for all $z \in\{r, t\}$, and $h\left(P_{N}\right)=x_{t}$ if either $x_{r} P_{j} x_{M(\{q, r, t\})} P_{j} x_{t}$ or $x_{t} P_{j} x_{M(\{q, r, t\})} P_{j} x_{r}$.

Note that $h$ is resolute by construction and is efficient as it refines the plurality rule. Moreover, it is anonymous, as $h\left(P_{N}\right)=h\left(P_{N}^{\prime}\right)$ whenever $P_{N}^{\prime}$ is anonymously equivalent to $P_{N}$. Since we have

$$
\begin{aligned}
& \#\left\{P_{N} \in \mathcal{T}_{\left\{x_{q}, x_{r}, x_{t}\right\}} \mid x_{M(\{q, r, t\})} P_{j} x_{z} \forall z \in\{r, t\}\right\} \\
& \quad=\#\left\{P_{N} \in \mathcal{T}_{\left\{x_{q}, x_{r}, x_{t}\right\}} \mid x_{z} P_{j} x_{M(\{q, r, t\})} \forall z \in\{r, t\}\right\} \\
& \quad=\#\left\{P_{N} \in \mathcal{T}_{\left\{x_{q}, x_{r}, x_{t}\right\}} \mid x_{r} P_{j} x_{M(\{q, r, t\})} P_{j} x_{t} \text { or } x_{t} P_{j} x_{M(\{t, q, r\})} P_{j} x_{r}\right\},
\end{aligned}
$$

$h$ is also $\mathrm{CN}$.

At this stage, one may be tempted to ask whether one can find an anonymous, $\mathrm{CN}$, efficient, and resolute SCR at any $(m, n)$. The following theorem advises caution on this.

Theorem 7 There exists a social choice problem which admits no anonymous, CN, efficient, and resolute SCR.

Proof Let $A=\{x, y\}$ and $N=\{1,2\}$. We have four possible profiles, $P_{N}, P_{N}^{\prime}, P_{N}^{\prime \prime}, P_{N}^{\prime \prime \prime}$ as shown below. 


$$
\begin{aligned}
& \frac{P_{1} P_{2}}{x x} \quad \frac{P_{1}^{\prime} P_{2}^{\prime}}{y \quad y} \quad \frac{P_{1}^{\prime \prime} P_{2}^{\prime \prime}}{x y} \quad \frac{P_{1}^{\prime \prime \prime} P_{2}^{\prime \prime \prime}}{y \quad x} \\
& \begin{array}{llllllll}
y & y & x & x & y & x & x & y
\end{array}
\end{aligned}
$$

Efficiency implies choosing $x$ at $P_{N}$ and $y$ at $P_{N}^{\prime}$. Moreover, $f\left(P_{N}^{\prime \prime}\right)=f\left(P_{N}^{\prime \prime \prime}\right)$ by anonymity. Hence, $\#\left\{P_{N} \in \mathcal{L}(A)^{N}: f\left(P_{N}\right)=x\right\} \neq \#\left\{P_{N} \in \mathcal{L}(A)^{N}: f\left(P_{N}\right)=y\right\}$, a failure of consequential neutrality.

Nevertheless, the social choice problem in the proof of Theorem 7 admits an anonymous, $\mathrm{CN}$, and resolute SCR. ${ }^{15}$ This raises the question of how general is the compatibility between anonymity and consequential neutrality when we dispense with the efficiency condition.

Moulin (1991) introduces the following condition that we call $\psi(m, n)$. Let $\mathcal{D}(n) \backslash\{1\}=\left\{d_{1}, \ldots, d_{K}\right\}$ for some $K \in \mathbb{N}$.

Condition $\psi(m, n): \nexists\left(a_{1}, \ldots, a_{K}\right) \in(\mathbb{N} \cup\{0\})^{K}$ such that $\sum_{i=1}^{K} a_{i} d_{i}=m$.

Remark $2 \mu(m, n) \Longrightarrow \psi(m, n)$ for all $n, m \geq 2$.

The following theorem states the cases of incompatibility of anonymity and neutrality in resolute social choice.

Theorem 8 (Moulin 1991) There exists an anonymous, neutral, and resolute SCR if and only if $\psi(m, n)$ holds.

We are now ready to state and prove our final theorem, which shows that if $m>n$, there exist anonymous, $\mathrm{CN}$, and resolute SCRs for any social choice problem with size $(m, n)$.

Theorem 9 For all social choice problems with $n<m$, there exists an anonymous, $C N$, and resolute $S C R$.

Proof Given any preference profile, we observe $k$ distinct preferences for some $k \in\{1, \ldots, \min \{m !, n\}\}$. There are $\left(\begin{array}{c}m ! \\ k\end{array}\right)$ ways to choose $k$ preferences from $\mathcal{L}(A)$. Let $\mathbf{P}=\left\{p^{1}, \ldots, p^{k}\right\}$ be a set of $k$ distinct preferences. Write $V^{k}$ for the set of vectors $v=\left(v_{1}, \ldots, v_{k}\right)$ with $v_{i} \geq 1$ for all $i \in\{1, \ldots, k\}$ and $\sum_{i=1}^{k} v_{i}=n$. Each $v \in V^{k}$, combined with $\mathbf{P}$, induces a set of profiles $E_{v}^{\mathbf{P}}$ that consists of all profiles where $p^{i}$ appears $v_{i}$ times for all $i \in\{1, \ldots, k\}$. Let $E^{\mathbf{P}}=\bigcup_{v \in V^{k}} E_{v}^{\mathbf{P}}$. Three remarks are in order. First, $\# E^{\mathbf{P}}$ depends on $k$ and not on the preferences in P. Second, an SCR $f$ that satisfies at any given $k \in\{1, \ldots, \min \{m !, n\}\}$ and $\mathbf{P}$ the invariance $f\left(P_{N}\right)=f\left(P_{N}^{\prime}\right)$ for all $P_{N}, P_{N}^{\prime} \in E^{\mathbf{P}}$ is anonymous. Third, $m \mid\left(\begin{array}{c}m ! \\ k\end{array}\right)$ if $k<m$, which is ensured

$\overline{15}$ To see this, consider $g: \mathcal{L}(A)^{N} \rightarrow \mathcal{A}$ such that $g\left(P_{N}\right)=g\left(P_{N}^{\prime}\right)=x$ and $g\left(P_{N}^{\prime \prime}\right)=g\left(P_{N}^{\prime \prime \prime}\right)=y$, which is both anonymous and $\mathrm{CN}$ while not efficient. 
when $n<m$. Now, let $t_{k}=\left(\begin{array}{c}m ! \\ k\end{array}\right) / m$. Write $A=\left\{x_{1}, \ldots, x_{m}\right\}$ and at each $k \in\{1, \ldots, \min \{m !, n\}\}$ assign to every $x_{i} t_{k}$ distinct sets $\mathbf{P}=\left\{p^{1}, \ldots, p^{k}\right\}$ and let $f\left(P_{N}\right)=x_{i}$ for all $P_{N} \in E^{\mathbf{P}}$ at every $\mathbf{P}$ assigned to $x_{i}$ for all $i \in\{1, \ldots, m\}$. By the three remarks, $f$ is anonymous, $\mathrm{CN}$, and resolute.

\section{Conclusion}

Using an irresolute SCR, one cannot reach a collective choice without referring to an additional mechanism that is external to the SCR. Therefore, Theorems 1 and 8 reflect the impossibility of making a collective choice by being confined to anonymous and neutral (efficient) SCRs as collective choice procedures. We take two different but related approaches to address how severe this impossibility is.

First, we identify the minimal irresoluteness outlook that would arise when anonymity, efficiency, and neutrality make ties inevitable. Based on this analysis, we deliver a method which, while preserving anonymity, efficiency, and neutrality, refines SCRs that deliver more ties than necessary.

Next, we introduce consequential neutrality as a weakening of neutrality. As expected, we obtain results that are more permissive than the (im-)possibilities announced by Theorems 1 and 8. We identify a large class of social choice problems where resoluteness becomes possible just because consequential neutrality replaces neutrality. Nevertheless, when efficiency is preserved, we know that this possibility does not hold for every social choice problem.

Dispensing with efficiency presents a case of interest. We show that anonymous, $\mathrm{CN}$, and resolute social choice is possible when $m>n$. Although this condition is logically independent of the necessary and sufficient condition of Theorem 8 , it opens the door of resoluteness to a large class of social choice problems that are doomed to irresoluteness by Theorem 8. Moreover, we are not able to find any social choice problem where anonymity, consequential neutrality, and resoluteness are incompatible. This provokes to ask whether these three conditions are compatible for any size of the social choice problem, which we leave as a-combinatorically difficult_open question.

\section{Appendix: Observations on the numbers of $\mathrm{CN}$, neutral, and resolute SCRs}

Tables 2 and 3 below show ratios the observations made in Sect. 4 are based on. 
Table 2 The ratio of $\# \mathcal{F}_{m, n}^{\text {NEUTRAL }} / \# \mathcal{F}_{m, n}^{C N}$ for different values of $(m, n)$

\begin{tabular}{llll}
\hline$\# \mathcal{F}_{m, n}^{\text {NEUTRAL }} / \# \mathcal{F}_{m, n}^{C N}$ & $n=2$ & $n=3$ & $n=4$ \\
\hline$m=2$ & 0.333333 & 0.114286 & 0.00994561 \\
$m=3$ & $3.58965 \times 10^{-14}$ & $5.73212 \times 10^{-85}$ & $\underline{\mathbf{0}}$ \\
$m=4$ & $\underline{\mathbf{0}}$ & $\underline{\mathbf{0}}$ & $\underline{\mathbf{0}}$ \\
\hline
\end{tabular}

$\underline{0}$ represents the values smaller than $10^{-10 m n}$

Table 3 The ratio of $\# \mathcal{F}_{m, n}^{C N} / \# \mathcal{F}_{m, n}^{\text {RESOLUTE }}$ for different values of $(m, n)$

\begin{tabular}{lllll}
\hline$\# \mathcal{F}_{m, n}^{C N} / \# \mathcal{F}_{m, n}^{\text {RESOLUTE }}$ & $n=2$ & $n=3$ & $n=4$ & $n=5$ \\
\hline$m=2$ & 0.75 & 0.546875 & 0.392761 & 0.2799 \\
$m=3$ & 0.135304 & 0.0229012 & 0.0038267 & 0.000638057 \\
$m=4$ & 0.00175989 & 0.0000149993 & $1.27583 \times 10^{-7}$ & $1.08512 \times 10^{-9}$ \\
$m=5$ & $8.19334 \times 10^{-7}$ & $5.69061 \times 10^{-11}$ & $3.95181 \times 10^{-15}$ & $\underline{\mathbf{0}}$ \\
$m=6$ & $8.12216 \times 10^{-12}$ & $\underline{\mathbf{0}}$ & $\underline{\mathbf{0}}$ & $\underline{\mathbf{0}}$ \\
\hline
\end{tabular}

$\underline{0}$ represents the values smaller than $10^{-10 m n}$

\section{References}

Bubboloni D, Gori M (2014) Anonymous and neutral majority rules. Soc Choice Welf 43(2):377-401

Bubboloni D, Gori M (2015) Symmetric majority rules. Math Soc Sci 76:73-86

Bubboloni D, Gori M (2016) Resolute refinements of social choice correspondences. Math Soc Sci $84: 37-49$

Campbell DE, Kelly JS (2015) The finer structure of resolute, neutral, and anonymous social choice correspondences. Econ Lett 132:109-111

Doğan O, Giritligil AE (2015) Anonymous and neutral social choice: Existence results on resoluteness. Tech Rep

King SS, Powers RC (2018) Beyond neutrality: extended difference of votes rules. Math Soc Sci 93:146-152

Moulin H (1980) Implementing efficient, anonymous and neutral social choice functions. J Math Econ 7(3):249-269

Moulin H (1983) The strategy of social choice. Number 18 in Advanced Textbooks in Economics. NorthHolland Pub. Co

Moulin H (1991) Axioms of Cooperative Decision Making. Number 15 in Econometric Society Monographs. Cambridge University Press

Perry J, Powers RC (2008) Aggregation rules that satisfy anonymity and neutrality. Econo Lett 100(1):108-110

Zwicker W (2016) Introduction to the theory of voting. In: Brandt F, Conitzer V, Endriss U, Lang J, Procaccia AD (eds) Handbook of Computational Social Choice, pages 23-56. Cambridge University Press

Publisher's Note Springer Nature remains neutral with regard to jurisdictional claims in published maps and institutional affiliations. 